\title{
HEMODINÂMICA GLOMERULAR RENAL NO ROEDOR CALOMYS CALLOSUS
}

\author{
Mirian A. Boim e Nestor Schor
}

\begin{abstract}
A função renal do roedor Calomys callosus, envolvido no ciclo de transmissão de diversos agentes patogênicos para o homem foi avaliada no animal intacto, através da técnica de depuraçāo e micropunçāo renal. Os resultados mostraram que este roedor apresenta niveis pressóricos, hematócrito e proteinas plasmáticas semelhantes aos dos ratos submetidos ao mesmo procedimento experimental. Os pesos corporal e renal, bem como a filtração glomerular global e por nefro assemelham-se aos do camundongo. Surpreendentemente estes roedores apresentaram significante número de glomérulos superficiais por rim, permitindo a avaliação da hemodinâmica glomerular. Apesar da pressão arterial semelhante à dos ratos Munich-Wistar $(M W)$, a pressão hidráulica intraglomerular no Calomys callosus foi inferior. Esta redução foi conseqüente à menor resistência pós-glomerular quando comparada à dos ratos $M W$. O fluxo plasmático glomerular atingiu valor bastante elevado em relação á filtração glomerular por nefro, fato que não só compensaria a reduzida pressão intraglomerular, como também seria suficiente para elevar a filtraçāo (por $\mathrm{g} / \mathrm{rim}$ ) a niveis superiores neste roedor, pois o coeficiente de ultrafiltração glomerular $(K f)$ foi semelhante ao do rato $M W$. O presente trabalho sugere que apesar das dificuldades técnicas que este animal impóe devido ao seu reduzido tamanho, o estudo da função renal global bem como da hemodinámica glomerular é factível, podendo portanto ser utilizado como modelo para estudo da função renal em doencas tropicais.
\end{abstract}

Palavras-chaves: Calomys callosus. Doenças tropicais. Função renal. Hemodinâmica glomerular. Microcirculação renal.

O pequeno roedor Calomys callosus Rengger 1830 (Rodentia Cricetidae) presente nas regiōes planas sul-americanas, destacando-se na Bolívia, Argentina e Brasil, apresenta caracteristicas relevantes para pesquisas em diversas áreas.

Semelhante ao camundongo ${ }^{12}$, este roedor possui a capacidade de participar no ciclo de transmissão de diversos microorganismos patogênicos para o homem, incluindo os agentes da febre hemorrágica, doença de Chagas, esquistossomose, leishmaniose, malária e leptospirose 148131416 .

A utilização do Calomys callosus tem sido proposta como modelo para o estudo dos mecanismos de imunotolerância bem como da dinâmica do processo infeccioso. Por outro lado, pouco se sabe sobre as funções fisiológicas deste roedor e, portanto, esta caracterização no animal intacto torna-se importante.

Desta forma, o intuito deste trabalho foi caracterizar a função renal do animal normal, ou seja, livre

Estudos realizados no Laboratório de Hemodinâmica Glomerular da Disciplina de Nefrologia da Escola Paulista de Medicina, financiado pela FAPESP, FINEP e IPEPENHI. Endereço para correspondência: prof. Dr. Nestor Schor, Disciplina de Nefrologia, Escola Paulista de Medicina, R. Botucatu, 720 - V. Clementino - 04023 São Paulo, SP.

Recebido para publicação em 3/8/88. de qualquer agente infeccioso, visando posteriormente utilizá-lo como modelo experimental no estudo dos efeitos de doenças tropicais sobre a função renal, especialmente sobre a hemodinâmica glomerular e a microcirculação renal.

\section{MATERIAL E MÉTODOS}

O animal foi cedido ao Biotério Central da Escola Paulista de Medicina pelo Biotério de Camundongos Isogênicos do Departamento de Imunologia do Instituto de Ciências Biomédicas da USP, onde iniciou-se a criaçāo de uma cepa de animais não contaminados pelos agentes patogênicos referidos.

Foram utilizados animais adultos, submetidos a estudos de função renal e hemodinâmica glomerular através das técnicas de depuração e micropunção, estabelecidas neste Serviço ${ }^{3}$.

Após serem pesados, os animais foram anestesiados com inactina, $100 \mathrm{mg} / \mathrm{kg}$, i.p. (Byk Gulden Konstanz, Alemanha). Em seguida foi realizada traqueostomia, cateterização da artéria femoral esquer$\mathrm{da}$, através de cateter (PE 10) para monitorização da pressão arterial média (PAM) (Gould Recorder 2200), bem como coleta de sangue, onde se determinou o hematócrito, concentração plasmática de proteinas (refratometria), inulina e ácido paraminohipurico $(\mathrm{PAH})^{7} 17$. 
A veia jugular esquerda foi cateterizada(PE 10) para a infusão de soluções de inulina a 10\% e PAH a $2 \%$ em soro fisiológico na velocidade de $2 \mu \mathrm{l} / \mathrm{min}$, bem como de soro homólogo na velocidade inicial de $5,2 \mu \mathrm{l} / \mathrm{min}$, para reposição das perdas cirúrgicas, ficando o animal em condições de euvolemia 11 . Procedeu-se à cateterização da bexiga (PE 50) para a coleta de urina em tubos graduados para a determinação do volume minuto e dosagem urinária de inulina e PAH 7 17. A filtração glomerular e o fluxo plasmático renal foram obtidos através das depurações de inulina e PAH, respectivamente. Adicionalmente, o rim esquerdo foi preparado para o emprego da técnica de micropunção ${ }^{3}$, que permite avaliar a função glomerular de nefros superficiais. Em resumo esta técnica consiste em coletar amostras de fluido tubular obtidas de túbulos proximais através de uma micropipeta com bisel em torno de 8 micras. Posteriormente, o volume foi determinado $2 \mathrm{e}$ a amostra destinou-se a dosagem da concentração de inulina através de método fluorimétrico 18 . Com este procedimento foi possivel a determinação da filtração glomerular por nefro (SNGFR).

Surpreendentemente, estes animais apresentaram significante número de glomérulos superficiais, o que permitiu a determinação de pressões hidráulicas

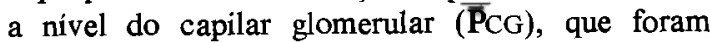
obtidas através de aparelho "Servo-Null", (modelo 4A, IPC, USA), que permite medidas de pressão hidráulica diretamente das microestruturas do rim.

Além do PCG foram obtidas as pressões hidráulicas de arteriolas eferentes superficiais (PAE), de capilares peritubulares (PC) e de túbulos proximais (PT), através de técnica utilizada neste Serviço ${ }^{3}$.

\section{RESULTADOS}

$\mathrm{Na}$ Tabela 1 estão expressos os valores individuais e as médias \pm erro padrão ( $\mathrm{X} \pm \mathrm{EP})$ do peso corporal (PC), peso do rim esquerdo (PR), pressão arterial média (PAM), hematócrito (Htc) e proteinas plasmáticas (Prot). O PC no animal adulto variou de 28 a 45 g sendo em média de $35 \pm 3 \mathrm{~g}$, e o PR foi em média de $0,19 \pm 0,03 \mathrm{~g}$. A PAM no animal sob anestesia com inactina manteve-se em niveis semelhantes aos obtidos em nosso Laboratório para ratos sob as mesmas condições, sendo em média $99 \pm 7 \mathrm{mmHg}$. O Htc e Prot apresentaram valores médios de $41 \pm 2 \%$ e $5,3 \pm 0,1 \mathrm{~g} / \mathrm{dl}$, respectivamente.

Tabela 1. Dados gerais do Calomys callosus

\begin{tabular}{|c|c|c|c|c|c|}
\hline $\mathrm{N}^{\circ}$ & PC & PR & $\begin{array}{l}\text { PAM } \\
\text { mmHg }\end{array}$ & $\begin{array}{c}\text { HTC } \\
\%\end{array}$ & $\begin{array}{c}\text { PROT } \\
\text { g/dl }\end{array}$ \\
\hline 1 & 35 & 0,21 & - & - & - \\
\hline 2 & 32 & 0,19 & - & - & - \\
\hline 3 & 28 & 0,14 & 105 & 43 & 5,1 \\
\hline 4 & 30 & 0,17 & 90 & 37 & 5,1 \\
\hline 5 & 40 & 0,20 & 115 & 41 & 5,6 \\
\hline 6 & 45 & 0,21 & 85 & 42 & 5,3 \\
\hline$\overline{\mathbf{X}}$ & 35 & 0,19 & 99 & 41 & 5,3 \\
\hline$\pm \mathrm{EP}$ & 3 & 0,03 & 7 & 2 & 0,1 \\
\hline
\end{tabular}

Tabela 2. Função tenal global de Calomys callosus

\begin{tabular}{|c|c|c|c|c|c|c|c|}
\hline \multirow[t]{2}{*}{ No } & $\begin{array}{c}V^{\prime} \\
\text { (2 rins) }\end{array}$ & $\begin{array}{c}V^{\prime} \\
(\mathrm{g} / \mathrm{rim})\end{array}$ & $\begin{array}{c}\text { FG } \\
(2 \text { rins })\end{array}$ & $\underset{(\mathrm{g} / \mathrm{rim})}{\mathrm{FG}}$ & $\begin{array}{c}\text { FPR } \\
(2 \text { rins })\end{array}$ & $\begin{array}{c}\text { FPR } \\
(\mathrm{g} / \mathrm{rim})\end{array}$ & FF \\
\hline & \multicolumn{3}{|c|}{..ul $/ \min .}$. & \multicolumn{3}{|c|}{ 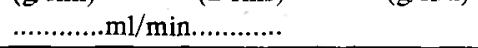 } & $\%$ \\
\hline 1 & 0,5 & 1,2 & 0,05 & 0,12 & 0,17 & 0,81 & 29 \\
\hline 2 & 0,4 & 1,1 & 0,06 & 0,16 & - & - & - \\
\hline 3 & 0,8 & 2,9 & 0,20 & 0,71 & 2,30 & 7,62 & 8 \\
\hline 4 & 2,6 & 7,6 & 0,23 & 0,68 & 1,66 & 4,91 & 10 \\
\hline 5 & 0,3 & 0,8 & 0,08 & 0,20 & - & - & - \\
\hline 6 & 2,0 & 4,8 & 0,17 & 0,40 & - & - & - \\
\hline$\overline{\mathbf{X}}$ & 1,1 & 3,1 & 0,13 & 0,38 & 1,38 & 4,44 & 17 \\
\hline$\pm \mathrm{EP}$ & 0,4 & 1,1 & 0,03 & 0,11 & 0,63 & 1,95 & 6 \\
\hline
\end{tabular}

Os dados de função renal global, ou seja, do rim como um todo, estão apresentados na Tabela 2 . O volume minuto (V'), a filtração glomerular global (FG) e o fluxo plasmático renal (FPR) foram expressos em valor absoluto ( 2 rins) bem como corrigidos por grama de rim. Os valores absolutos para V', e FG FPR fo- 
ram em média de $1,1 \pm 0,4 \mu \mathrm{l} / \mathrm{min}, 0,13 \pm 0,03 \mathrm{ml} / \mathrm{mine}$ $1,38 \pm 0,63 \mathrm{ml} / \mathrm{min}$, respectivamente, e quando corrigidos por grama de tecido renal, $3,1 \pm 1,1 \mu \mathrm{l} / \mathrm{min}$, $0,38 \pm 0,11 \mathrm{ml} / \mathrm{min}$ e $4,44 \pm 1,95 \mathrm{ml} / \mathrm{min}$, respectivamente.

O fluxo plasmático renal apresentou valores elevados em relação ao que foi filtrado (FG), resultando em uma fração de filtração (FF) relativamente menor $(17 \pm 6 \%)$ nestes animais, quando comparada com outros mamiferos.

A filtração glomerular por nefro (SNGFR) obtida através de micropunção de nefros superficiais
(Tabela 3) foi em média de $9,15 \pm 1,45 \mathrm{nl} / \mathrm{min}$, valor reduzido quando comparado com outras especies. Porém, quando corrigido por grama de rim $(46,57 \pm 7,61$ $\mathrm{nl} / \mathrm{min} / \mathrm{g} \mathrm{rim}$ ) mostrou-se maior ao observado para o rato Munich-Wistar (MW), em condições euvolêmicas (Tabela 4 e 5). As pressões hidráulicas obtidas por punção direta das microestruturas superficiais do rim mostraram valores médios para $\overline{\mathrm{P}}_{\mathrm{CG}}$ de $35 \pm 1 \mathrm{mmHg}$, PT de $9 \pm 1 \mathrm{mmHg}$, PAE de $16 \pm 2 \mathrm{mmHg}$ e para PC de $5 \pm 1 \mathrm{mmHg}$. A pressão hidráulica transglomerular $(\overline{\Delta \bar{P}})$, obtida através da diferença entre $\overline{\mathrm{P}}_{\mathrm{CG}}$ e PT foi em média de $26 \mathrm{mmHg}$.

Tabela 3 - Hemodinâmica glomerular de Calomys callosus

\begin{tabular}{|c|c|c|c|c|c|c|}
\hline & SNGFR & $\begin{array}{r}\text { SNGFR } \\
\mathrm{g} / \mathrm{rim}\end{array}$ & $\overline{\mathbf{P}}$ CG & $\mathrm{Pt}$ & P ae & $P_{c}$ \\
\hline & $\begin{array}{r}7,84 \\
9,46 \\
11,51 \\
5,15 \\
5,44 \\
10,59 \\
11,66 \\
3,15 \\
17,57\end{array}$ & $\begin{array}{l}37,33 \\
45,05 \\
54,81 \\
27,11 \\
28,63 \\
55,64 \\
61,38 \\
16,58 \\
92,48\end{array}$ & $\begin{array}{l}34 \\
37 \\
34 \\
30 \\
28 \\
36 \\
34 \\
34 \\
38 \\
40\end{array}$ & $\begin{array}{r}10 \\
10 \\
10 \\
12 \\
10 \\
12 \\
10 \\
8 \\
10 \\
6 \\
11 \\
9 \\
8 \\
6\end{array}$ & $\begin{array}{l}12 \\
10 \\
13 \\
14 \\
24 \\
20 \\
22\end{array}$ & $\begin{array}{l}8 \\
5 \\
4 \\
2 \\
5 \\
6 \\
6\end{array}$ \\
\hline$\underset{ \pm E P}{X}$ & 9,15 & 46,57 & 35 & 9 & 16 & 5 \\
\hline
\end{tabular}

Tabela 4 - Dados comparativos entre Calomys callosus e outros roedores

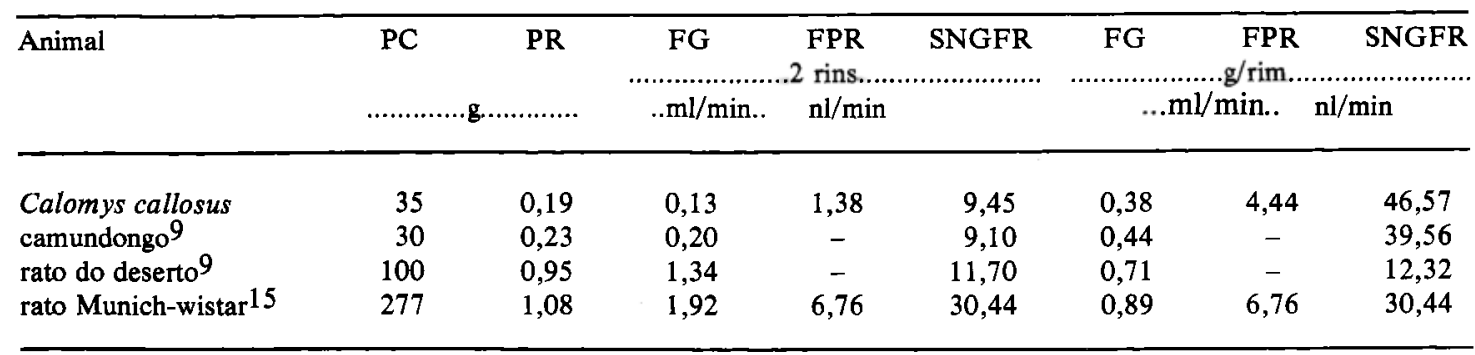

A Tabela 4 apresenta dados comparativos entre - Calomvs callosus, camundongo ${ }^{15}$, rato do deserto ${ }^{15}$ e rato Munich-wistar ${ }^{9}$. Os resultados mostram que o Calomys callosus se assemelha ao camundongo, tanto em relação aos pesos corporal e renal, quanto a função renal global e por nefro, ressaitando que a SNGFR quando corrigida por grama de rim, tanto no Callomys callosus quanto no camundongo é maior do que os valores obtidos para o rato $\mathrm{MW}$. 
$\mathrm{Na}$ Tabela 5 apresentamos dados comparativos de hemodinâmica glomerular entre o Calomys callosus e rato MW. Com relação as pressões hidráulicas, os valores obtidos para todas as estruturas foram inferiores aos observados para o rato $\mathrm{MW}$, destacando-se a PCG, que foi em média, $10 \mathrm{mmHg}$ menor, salientando que esta diferença é bastante significativa, resultando em um $\overline{\Delta \mathbf{P}}$ também inferior, sendo $26 \mathrm{mmHg}$ no Calomys callosus e $31 \mathrm{mmHg}$ no rato $\mathrm{MW}$.

Embora tenha sido possivel a determinação de alguns parâmetros da hemodinâmica glomerular no Calomys callosus, em especial pela presença de glomérulos superficiais, a determinação de todos os parâmetros não foi possível devido às dificuldades técnicas envolvendo principalmente o tamanho do animal e das estruturas a serem micropuncionadas.
(FPR/22.000) foi de $63 \mathrm{nl} / \mathrm{min}$ e de $332 \mathrm{nl} / \mathrm{min}$ quando corrigido por grama de rim. A semelhança do que ocorre para oFPR, o QA atinge um valor bastante elevado em relação a SNGFR resultando em uma fração de filtração por nefro(SNFF) diminuida (14\%) quando comparada com outros mamiferos, como por exemplo o rato MW que possui SNFF de 30\% (Tabela 5). Por outro lado a SNFF calculada (14\%) é bastante semelhante à fração de filtração global(FG), que como apresentado foi em média de $17 \%$, neste roedor.

A partir da estimativa do QA e da SNFF e, através de cálculos matemáticos usualmente utilizados, determinamos os outros parâmetros envolvidos no processo de filtração glomerular. Assim, considerou-se que a SNGFR $=K_{f}$. PUF, onde PUF é a pressão de ultrafiltração, ou seja, PUF $=\overline{\Delta P}, \pi C G$, onde $\pi C G$ é a variação de pressão oncótica intraglome-

Tabela 5 - Hemodinâmica glomerular: dados comparativos entre Calomys callosus e rato Munich-Wistar

\begin{tabular}{|c|c|c|}
\hline & Calomys callosus & Munich-wistar \\
\hline SNGFR (nl/min) & 9,15 & 30,44 \\
\hline $\mathrm{SNGFR}(\mathrm{nl} / \mathrm{min} / \mathrm{g} \mathrm{rim})$ & 47,57 & 30,44 \\
\hline $\left.\mathrm{QA}^{\mathrm{A}} \mathrm{n} / \mathrm{min}\right)$ & 63 & 103 \\
\hline $\mathrm{QA}(\mathrm{nl} / \mathrm{min} / \mathrm{g} \mathrm{rim})$ & 332 & 103 \\
\hline SNFF (\%) & 14 & 30 \\
\hline$\overline{\mathrm{P}}_{\mathrm{CG}}(\mathrm{mmHg})$ & 35 & 45 \\
\hline PT (mmHg) & 9 & 14 \\
\hline$\overline{\Delta P}, \mathrm{mmHg})$ & 26 & 31 \\
\hline PAE (mmHg) & 16 & 19 \\
\hline $\mathrm{PC}(\mathrm{mmHg})$ & 5 & 8 \\
\hline RA $\left(10^{10}\right.$.din.s. $\left.\mathrm{cm}^{-5}\right)$ & 0,92 & 2,79 \\
\hline $\operatorname{RE}\left(10^{10}\right.$ din.s.cm $\left.{ }^{-5}\right)$ & 0,30 & 1,52 \\
\hline $\mathrm{RT}\left(10^{10}\right.$,din.s.cm $\left.{ }^{-5}\right)$ & 1,22 & 4,31 \\
\hline $\mathrm{Kf}(\mathrm{nl} / \mathrm{s} \cdot \mathrm{mmHg})$ & 0,1174 & 0,0860 \\
\hline
\end{tabular}

Neste sentido, paràmetros como a concentração protéica da arteriola eferente $(\mathrm{CE})$ não puderam ser determinados diretamente. Entretanto, o fluxo plasmatico glomerular $(\mathrm{QA})$ estimado pode ser calculado admitindo-se que o Calomys callosus possua numero de nefros semelhante ao camundongo (22.000 nefros $/ 2$ rins) ${ }^{15}$. Neste caso, o QA calculado rular, determinada pela integral desenvolvida por Deen e cols 6 . Para isto, utilizou-se a média da pressão oncótica da artériola aferente $(\pi A)$ e da eferente $(\pi E)$, que por sua vez são determinadas pelas concentrações da proteina $\mathrm{CA}$ e CE. Então, se a $\mathrm{SNFF}=1-\mathrm{CA} / \mathrm{CE}$, e a $\mathrm{CE}=\mathrm{CA} / 1-\mathrm{SNFF}$, determinamos que a $\mathrm{CE}=$ $6,2 \mathrm{~g} / \mathrm{dl}$. Portanto a $\pi \mathrm{E}$ é de $21,3 \mathrm{mmHg}$. Como a re- 
lação $\overline{\mathrm{P}}, / \pi \mathrm{E}$ foi maior do que 1 , caracterizamos situação hemodinâmica glomerular de desequilibrio, ou seja, ao longo das alças capilares do glomérulo, a pressão hidráulica sempre é maior que a oncótica, ocorrendo fenômeno de ultrafiltração. Esta situação de desequilibrio em euvolemia é semelhante àquela observada em ratos MW. Desta maneira é possivel utilizar o modelo matemático desenvolvido por Deen e cols ${ }^{6}$, possibilitando o cálculo dos outros parâmetros envolvidos na dinâmica glomerular. Assim, pode-se obter os valores estimados das resistências das arteriolas aferente (RA), eferente (RE) e total (RT), que foram $0,92,0,30$ e $1,22 \times 10^{10}$.din.s.cm ${ }^{-5}$, respectivamente. Estes valores sāo inferiores aos observados para ratos MW em nosso laboratório (Tabela 5). Por outro lado, o coeficiente de ultrafiltração glomerular $\left(\mathrm{K}_{\mathrm{f}}\right)$ no Calomys callosus $(0,1174 \mathrm{nl} / \mathrm{s} \cdot \mathrm{mmHg})$ foi semelhante ao observado para ratos $\mathrm{MW}(0,0860 \mathrm{nl} /$ $\mathrm{s} / \mathrm{mmHg}$ ).

Portanto, ressaltamos que nestes experimentos, somente a CE foi estimada, sendo os demais parâmetros calculados, segundo os modelos matemáticos vigentes para o estudo da hemodinâmica glomerular nos outros mamíferos.

\section{DISCUSSÃO}

O interesse no estudo do Calomys callosus, bem como a caracterização da sua função renal advém do fato deste roedor participar do ciclo de transmissão de várias doenças tropicais, desconhecendo-se em qual delas este animal poderia, além de ser reservatório destas patologias, adquirir a doença. Este aspecto é de importância pois os modelos experimentais de doenças tropicais para o estudo da função renal em ratos ou no cão, até o presente momento, são inadequados. Mais ainda, esta situação gera um desconhecimento significante das patologias nefrológicas tropicais, comuns no nosso meio, como a nefropatia da malária, esquistossomose, leptospirose, etc. Portanto, o presente trabalho pretende iniciar, sistematicamente, a busca de modelos experimentais acessiveis ao estudo da função renal em animais passíveis de serem infectados com estes agentes de patologias tropicais.

O estudo realizado mostrou que pelo menos dois aspectos podem ser ressaltados. Primeiro, é que embora este animal seja bastante pequeno e de manuseio relativamente dificil no que diz respeito aos procedimentos cirúrgicos (cateterização das estruturas), ele suporta a agressão da anestesia, cirurgia, bem como o tempo da duração do experimento $(\sim 4$ horas), sendo portanto factivel a sua utilização neste tipo de manobra experimental. $O$ outro aspecto que despertou interesse foi o fato deste roedor apresentar grande número de glomérulos superficiais ( $\sim 12$ por rim), característica que permite a avaliação quase completa de toda a hemodinâmica glomerular. Vale a pena salientar que esta característica descoberta inicialmente em uma colônia de ratos Wistar mutantes, denominados Munich-wistar (MW), proporcionou importante progresso no entendimento da fisiologia renal, pelo fato de permitir o estudo detalhado da função glomerular ${ }^{5}$. Sabe-se que em geral os mamiferos apresentam poucos glomérulos superficiais sendo que os ratos MW e os macacos ${ }^{10}$ são os que melhor fornecem esta disposição anatômica.

Com relação à função renal global e glomerular do Calomys callosus, vários aspectos interessantes foram observados no presente estudo. $O$ processo de filtração quando analisado para o rim total (FG) ou para o nefro único (SNGFR) comportou-se de forma diferente quando considerado como percentual dos valores obtidos para os ratos MW, ou seja, a SNGFR do Calomys callosus foi $15 \%$ do valor observado nos $\mathrm{MW}$, enquanto a $\mathrm{FG}$ atingiu um percentual de apenas $6 \%$. Algumas justificativas poderiam ser apresentadas para explicar a SNGFR relativamente maior do que a FG. Estes roedores poderiam apresentar um aumento na função glomerular de nefros superficiais em relação aos nefros médio corticais e justamedulares que teriam esta função diminuída, contribuindo para a FG mais baixa. Uma outra hipotese seria a de que estes roedores apresentassem reduzido número de nefros superficiais quando comparado com os nefros justamedulares, propiciando uma hiperfunção dos nefros superficiais, porem não em número suficiente para elevar a filtração renal global. Finalmente, a possibilidade de erro na coleta da urina através da bexiga não pode ser afastada, levando-se em conta o espaço morto que contribuiria de maneira significante para erros de coleta e determinação da função renal global. Nos ratos MW, bem como em espécies animais de maior porte, a coleta de urina é feita através da cateterização do ureter, eliminando-se este tipo de erro. Entretanto, no Calomys callosus, este procedimento não foi possivel pelo fato de se tratar de uma estrutura muito pequena e frágil.

Por outro lado, os parâmetros obtidos da hemodinàmica glomerular mostraram que o Calomys callosus apresenta valores de pressão hidráulica das microestruturas do rim inferiores aos observados para ratos MW (Tabela 5), sendo que a diferença mais importante foi observada com relação à pressão hidráulica intraglomerular ( $(\overline{\mathbf{P}} \quad \mathrm{CG})$. Este dado é intrigante, visto que a pressão arterıal sıstemica é semelhante entre ratos MW e o Calomys callosus (Tabela 1) e deveria ser transmitida ao glomérulo, resultando em uma PCG semelhante entre os dois roedores. Portanto, existe a possibilidade de que a pressão sistêmica não está sendo transmitida de forma adequada ao glomérulo, ou seja, que a resistência pré-glomerular (RA) 
seja maior neste roedor do que nos MW. Entretanto, os valores das resistências arteriolares calculadas indicam que a P $\mathrm{CG}$ não se mantém nos mesmos níveis dos obtidos nos MW devido à resistência eferente ser proporcionalmente menor (20\%) no Calomys callosus em relação aos ratos $\mathrm{MW}$, quando comparada com o valor da RA (33\%). A pesar de menor $\mathrm{PT}$, a redução da PCG no Calomys callosus contribuiu de forma significante para a diminuição da pressão hidráulica transglomerular $\left(\overline{\Delta \mathbf{P}}=\overline{\mathbf{P}}_{\mathrm{CG}}-\mathrm{PT}\right)$, a qual expressa a força hidráulica efetiva que favorece o processo da ultrafiltração. Por outro lado, o fluxo plasmático glomerular (QA) apresentou valor elevado em relação à filtração glomerular por nefro (SNGFR) implicando na diminuição da fração de filtração por nefro (SNFF), à semelhança do que ocorreu para a FF por rim total.

Utîilzando os cálculos matemáticos referidos, pudemos determinar o valor do $\mathrm{K}_{\mathrm{f}}$, sendo semelhante, em média, ao obtido para os ratos $\mathrm{MW}$.

Ao considerarmos os determinantes da filtração glomerular que são a $\overline{\Delta \mathrm{P}}, \mathrm{QA}, \mathrm{CA}$ e o $\mathrm{K}_{\mathrm{f}}$, podemos sugerir que em média a $\mathrm{CA}_{\mathrm{e}}$ o $\mathrm{K}_{\mathrm{f}}$ dos Calomys callosus são semelhantes aos ratos MW. Apesar de menor pressão hidráulica transglomerular $(\overline{\mathbf{A P}}, \quad$ a filtração glomerular por nefro $(\mathrm{g} / \mathrm{rim})$ é substancialmente maior nestes pequenos roedores às custas de reduzidas resistências intra-renais ( $R A$ e $R E$ ) que propiciariam aumento do fluxo plasmático glomerular (QA). Portanto, o elevado fluxo plasmático glomerular no Calomys seria o responsável pela maior filtração glomerular por nefro determinada.

Finalmente, utilizando esta metodologia de micropunção, pudemos avaliar o fenômeno da hemodinâmica glomerular e a microcirculação renal deste roedor, o qual enfatizamos ser um modelo importante para a pesquisa básica, onde não só os mecanismos imunológicos podem e devem ser estudados, mas também os mecanismos fisiopatológicos das doenças tropicais que comprometem a função renal podem, potencialmente, ser desenvolvidos.

\section{SUMMARY}

Renal function was characterized in Calomys callosus, a rodent which can participate in the transmission of several human diseases. The results showed that the pressures levels, hematocrit and plasmatic proteins were similar to rats submitted to the same experimental maneuvers. The corporal and renal weights, whole and single nephron glomerular flltration rates were similar to the mouse. This rodent presented a significant number of superficial glomeruli, allowing to evaluate glomerular hemodynamics and renal microcirculation parameters. Despite mean arterial pressure similar to the Munich-Wistar
(MW) rats, mean glomerular capillary hydraulic pressure was lower in Calomys callosus due to $a$ lower post glomerular resistance in this rodent when compared to $M W$ rats. The mean glomerular plasma flow rate was relatively elevated when compared with single nephron glomerular filtration rate, which in turn blunted the lower intraglomerular hydraulic pressure, and thus increased the single nephron glomerular filtration rate perg/kidney. The present work suggests that despite of the technical difficulties imposed by the reduced size of the Calomys callosus, the study of the renal function and glomerular hemodynamics is possible, establishing therefore, a modelfor the study such renal function.

Key-words: Calomys callosus. Renal function. Glomerular hemodynamics. Renal microcirculation.

\section{AGRADECIMENTOS}

Agradecemos aos responsáveis pelo Biotério Central da Escola Paulista de Medicina, especialmente à Dra. Adela Rosenkranz e à Miriam A. Ghiraldini, pelo fornecimento do Calomys callosus.

\section{REFERÊNCIAS BIBLIOGRÁFICAS}

1. Almeida CR. Relatório das Pesquisas do Plano de Peste de Exu. Agência Magalhães, Ministério da Saúde, 1973.

2. Andreucci VE. Calibrations. In Manual of renal micropuncture. Ed.: Vittorio E. Andreucci, Naples, pp.283287, 1978.

3. Barros EJG, Boim MA, Ajzen H, Ramos OL, Schor N. Glomerular hemodynamics and hormonal participation on cyclosporine nephrotocixity. Kidney International 32: $19-25,1987$

4. Borba CE. Infeção natural e experimental de alguns roedores pelo Schistosoma mansoni, Sambom, 1907. Tese de Mestrado, Universidade Federal de Minas Gerais, 1972.

5. Brenner BM, Troy JL, Daugharty TM. The dynamics of the glomerular ultrafiltration in the rat. Journal of Clinical Investigation 50: 1776-1780, 1971.

6. Deen WM, Troy JL, Robertson CR, Brenner BM. Dynamics of glomerular ultrafiltration in the rat: determination of the ultrafiltration coefficient. Journal of Clinical Investigation 52: 1500-1508, 1973.

7. Fuhr J, Kaczmarczyk J, Kruttgen CD. Eine einfache colorimetrische method zur inulin bestimmung for nierenclear anceuntersuchungen bei soffwechselgesuden and diabetibern. Klinical Wochenschrurt 33: 729-730, 1955.

8. Johnson KM, Mackenzie RB, Webb PA, Kuns ML Chronic infection of rodents by machupo virus. Science 150: 1618-1619, 1975.

9. Lugon JR. Função renal e hemodinâmica glomerular na endotoxemia experimental: participação do sistema re- 
Boim MA, Schor N. Hemodinâmica glomerular no roedor Calomys callosus. Revista da Sociedade Brasileira de Medicina Tropical 22: 31-37, jan-mar, 1989.

nina angiotensina, prostaglandina e vasopressina. Tese de Doutoramento, Escola Paulista de Medicina, 1986.

10. Maddox DA, Deen WM. Dynamics of glomerular ultrafiltration: Studies in the primates. Kidney International 5: 271-278, 1974 .

11. Maddox DA, Price DC, Rector FC Jr. Effects of surgery on plasma volume and salt and water excretion in rats. American Journal of Physiology 233:600-606, 1977.

12. Massola E, Fornes A. Nuevos dados sobre la morfologia distribucion geografica y etoecologia de Calomys callosus (Rengger) (Rodentia Cricetidae). Physis 25: 325, 1965.

13. Mello DA. Infecção experimental de Calomys callosus (Rengger, 1830), (Cricetidae-Rodentia) a quatro espécies de parasitos. Revista da Sociedade Brasileira de Medicina Tropical 13: 101-105, 1979/80.
14. Mello DA. Aspectos do ciclo silvestre do Trypanosoma cruzi em regiōes de cerrado (municipio de Formosa, Estado de Goiás). Memorias do Instituto Oswaldo Cruz 76: 227-246, 1981.

15. Renkin EM, Gilmore JP. Glomerular filtration. In Handbook of Physiology (Renal Physiology), Ed: Stephen R. Geiger, pp. 185-248, 1973.

16. Ribeiro RD. Novos reservatórios do Trypanosoma cruzi. Revista Brasileira de Biologia 33: 429-536, 1973.

17. Smith HW, Finkelstein N, Aliminosa L, Crawford B, Graber $\mathbf{M}$. The renal clearances of substituted hippuric acid derivatives and other aromatic acids in dog and men. Journal of Clinical Investigation 24: 388-404, 1945.

18. Vurek GG, Pegran SE. Fluorimetric method for the determination of nanograms quantities of inulin. Annals of Biochemistry 16: 409-419, 1966. 